\title{
Octreotide Acetate Long-Acting Formulation Versus Open-Label Subcutaneous Octreotide Acetate in Malignant Carcinoid Syndrome
}

\author{
By Joseph Rubin, Jaffer Ajani, W illiam Schirmer, Alan P. Venook, Ronald Bukowski, Rodney Pommier, Leonard Salt, \\ Paresh Dandona, and Lowell Anthony
}

\begin{abstract}
Purpose: Subcutaneous (SC) octreotide acetate effectively relieves the diarrhea and flushing associated $w$ ith carcinoid syndrome but requires long-term multiple injections daily. A microencapsulated long-acting formulation (LAR) of octreotide acetate has been developed for once-monthly intramuscular dosing.

Patients and Methods: A randomized trial compared double-blinded octreotide LAR at 10, 20, and $30 \mathrm{mg}$ every 4 weeks with open-label SC octreotide every 8 hours for the treatment of carcinoid syndrome. Seventynine patients controlled with treatment of SC octreotide 0.3 to $0.9 \mathrm{mg} / \mathrm{d}$ whose symptoms returned during a w ashout period and who returned for at least the w eek 20 evaluation constituted the efficacy-assessable population.

Results: Complete or partial treatment success was comparable in each of the four arms of the study (SC,
\end{abstract} $58.3 \%$; $10 \mathrm{mg}, 66.7 \%$; $20 \mathrm{mg}, 71.4 \%$; $30 \mathrm{mg}, 61.9 \%$;

$\mathbf{T}$ HE MOST COMMON symptoms of carcinoid tumors are collectively referred to as the carcinoid syndrome, which is associated with tumor secretion of excessive amounts of major regulatory peptides and amines, such as serotonin and its metabolites. Malignant carcinoid syndrome does not develop until a tumor has metastasized, often to the liver, and the hormonal products released by the tumor reach the systemic circulation in substantial concentrations. The principal signs and symptoms of carcinoid syndrome include sustained flushing (approximately 80\%), diarrhea (approximately 76\%), abdominal pain/cramping (approximately 70\%), endocardial fibrosis (approximately 20\%), ${ }^{1,2}$ and asthma-like symptoms or wheezing (10\% to $20 \%){ }^{3}$ Carcinoid tumors occur at an annual rate from less than 1 to

From the Mayo Clinic, Rochester, MN; M.D. Anderson Cancer Center, Houston, TX; the Ohio State University Hospital, Columbus, $\mathrm{OH}$; University of California at San Francisco, San Francisco, CA; The Cleveland Clinic Foundation, Cleveland, OH; Oregon Health Sciences University, Portland, OR; Memorial Sloan-Kettering Hospital, New York, NY; the State University of New York, Buffalo, NY; and Louisiana State University Medical Center, New Orleans, LA.

Submitted June 1, 1998; accepted September 30, 1998.

Supported by Novartis Pharmaceuticals Corp., East Hanover, NJ.

Address reprint requests to Joseph Rubin, MD, Mayo Clinic, Division

of Medical Oncology, 200 First St, SW, Rochester, MN 55905.

(1) 1999 by American Society of Clinical Oncology.

0732-183X/99/1702-0600\$3.00/0
$P \geq .72$ for all pairwise comparisons). Control of stool frequency was similar in all treatment groups. Flushing episodes were best controlled in the 20-mg LAR and SC groups; the 10-mg LAR treatment was least effective in the control of flushing. Treatment was well tolerated by patients in all four groups.

Conclusion: Once octreotide steady-state concentrations are achieved, octreotide LAR controls the symptoms of carcinoid syndrome at least as well as SC octreotide. A starting dose of $\mathbf{2 0} \mathbf{~ m g}$ of octreotide LAR is recommended. Supplemental SC octreotide is needed for approximately $\mathbf{2}$ w eeks after initiation of octreotide LAR treatment. Occasional rescue SC injections may be required for possibly 2 to 3 months until steady-state octreotide levels from the LAR formulation are achieved.

J Clin Oncol 17:600-606. @ 1999 by American Society of Clinical Oncology.

2.1 per 100,000 in England ${ }^{4}$ and the United States. ${ }^{5-9}$ The subsequent development of carcinoid syndrome depends on the site of origin of the tumor, total tumor mass, and the extent of metastasis. $^{2}$

Octreotide acetate is a synthetic octapeptide that has a biologic profile similar to endogenous somatostatin but has a longer half-life and duration of activity that allow treatment with subcutaneous (SC) administration two to four times daily. ${ }^{10}$ This short-acting formulation of octreotide administered subcutaneously in multiple daily doses is indicated for the treatment of acromegaly and for symptomatic control of vipomas and carcinoid tumors. Octreotide inhibits secretion of bioactive substances (eg, serotonin, tachykinins) that cause the symptoms of carcinoid syndrome. ${ }^{8,11-14}$ A longacting octreotide formulation (octreotide acetate LAR), consisting of microspheres of poly-DL-lactide-co-glycolideglucose containing octreotide, has been developed with the goal of improving patient convenience, compliance, and quality of life by providing similar therapeutic benefits with significantly less discomfort and inconvenience. Octreotide LAR exhibits all the characteristics of the short-acting SC formulation and has the added advantage of slow drug release, which occurs by the cleavage of the polymer ester linkage, primarily through tissue fluid hydrolysis. Dosing is recommended every 4 weeks. Doses of up to $60 \mathrm{mg}$ of octreotide LAR have been shown to be well tolerated in healthy volunteers and to be an effective treatment in 
patients with acromegaly. ${ }^{15,16}$ This study, the first controlled trial of octreotide LAR for the treatment of carcinoid syndrome, was designed to evaluate the efficacy over a 6-month period of multiple dose levels of octreotide LAR compared with SC octreotide in providing continuous symptomatic control of malignant carcinoid syndrome when given at 4-week intervals. Secondary objectives were to assess safety and tolerability, dose proportionality of octreotide serum concentrations, and excretion of the serotonin metabolite 5-hydroxyindoleacetic acid (5-HIAA). Because of the low incidence and prevalence of carcinoid syndrome, accrual to achieve statistical significance between treatment groups was not a goal of the study design.

\section{PATIEN TS AND METHODS}

\section{Study Design}

This prospective multicenter trial was a parallel-group design. Patients experienced control of symptoms when treated with SC octreotide acetate (Sandostatin; Novartis Pharmaceuticals Corp., East Hanover, NJ). Patients continued to show symptom control for at least a 2-week screening period, which was followed by a washout period of 3 to 5 days. After washout, patients were randomly assigned to receive one of four treatments: either $10 \mathrm{mg}, 20 \mathrm{mg}$, or $30 \mathrm{mg}$ of octreotide acetate LAR (Sandostatin LAR; Novartis) administered intramuscularly by medical personnel, or continuation of SC octreotide every 8 hours at the same dosage received during screening. Assignment to the three doses of octreotide LAR was double-blind. All patients in the octreotide LAR groups received an initial dose on day 1 . Because of the kinetics of octreotide release and time to achieve therapeutic concentrations of octreotide from the LAR formulation, patients randomized to any of the octreotide LAR groups continued to receive concomitant SC octreotide every 8 hours at their previous dosage through day 11 . Subsequent intramuscular injections of octreotide LAR were given at the end of weeks $4,8,12,16$, and 20.

\section{Patients}

Men and nonpregnant, nonlactating women at least 18 years of age with a histologically confirmed diagnosis of carcinoid tumor with carcinoid syndrome were eligible for enrollment. Symptoms of flushing and diarrhea had to have been well controlled by SC octreotide, ie, two or fewer flushing episodes per day and an average stool frequency of three or fewer per day (with a maximum of five stools on any 1 day) during a 2-week screening period during which patients received 0.3 to $0.9 \mathrm{mg}$ SC octreotide per day. For patient efficacy to be assessable, symptoms must have returned (three episodes of flushing in a single day and/or an increase of at least two stools daily for 2 consecutive days above the prewashout average) during a washout period of 3 to 5 days. Women with childbearing potential were required to use effective, medically approved contraception throughout the study. Patients were excluded for any of the following reasons: the likelihood of receiving additional medical therapy for carcinoid syndrome (eg, surgery, chemotherapy, radiation therapy, interferon) that might alter symptoms; the presence of any intercurrent medical condition that might interfere with the conduct or evaluation of study results or that might affect the interpretation of clinically relevant laboratory values; symptomatic abnormalities of the biliary tract or diarrhea from causes other than carcinoid syndrome, or a history of alcohol or drug abuse in the previous 6 months; unwillingness to follow dietary restrictions within 3 days of urinary 5-HIAA sample collection or need for medications (eg, reserpine, mephenesin carbamate, Lugol's solution) that would interfere with urinary 5-HIAA measurement; or inability to return for all required evaluations. The study was reviewed and approved by the institutional review boards of all participating institutions, and all patients gave written informed consent before entering the washout period.

\section{Procedures}

Patients receiving intramuscular octreotide LAR who experienced a loss of symptomatic control were permitted to use rescue SC octreotide, which was self-administered. If stool frequency increased by at least two stools per day for 2 consecutive days above the screening daily average, or if flushing frequency increased to at least three episodes per day for 1 day, rescue doses of SC octreotide every 8 hours were permitted until symptoms were controlled to screening frequency. Patients randomized to receive SC octreotide who required rescue could increase their SC dosage by $50 \%$; patients receiving LAR could begin SC rescue doses at the same dosage used before washout. Once symptom relief was achieved for 24 hours, the patients were instructed to discontinue rescue therapy. Rescue could be repeated a second time. In the event the first two episodes required more than 5 days total of rescue medication or if a third rescue episode was needed, patients continued to receive SC rescue medication until the next regularly scheduled visit.

\section{Efficacy and Safety Assessments}

The primary efficacy assessment was treatment response, which was defined as either treatment success, partial treatment success, or treatment failure based on the degree and duration of suppression of carcinoid symptoms, as indicated by the need for SC rescue therapy by patients randomized to one of the octreotide LAR groups or the need for an increase in dosage in patients randomized to the SC octreotide group. Treatment response was further defined as follows: treatment success, symptomatic control without the need for rescue medication at any time point; partial success, partial control with rescue needed on no more than two occasions for not more than 5 days total during the intervals of treatment weeks 17 to 20 and 21 to 24 ; and treatment failure, rescue required on three or more occasions or for 5 days or more during weeks 17 to 20 and 21 to 24 . Final assessments of treatment response were made after the fifth and sixth doses (weeks 20 and 24), by which time steady-state octreotide levels could be expected in the groups receiving octreotide LAR.

Efficacy assessments included the daily frequency of stools and flushing episodes, number of patients using rescue medication, and 24-hour urinary 5-HIAA concentrations. Patients recorded clinical symptoms and any rescue therapy in daily diaries that were submitted weekly.

For evaluation of 24-hour urinary 5-HIAA, samples were taken at screening, baseline, and weeks 4, 8, 12,16, 20, and 24. Serum octreotide levels were measured only for patients receiving any one of the three doses of octreotide LAR at baseline and at weeks 4, 8, 12, 16, 20, and 24. Because the kinetics of SC octreotide were previously determined, octreotide levels were not measured in patients receiving SC octreotide.

Safety assessments, including physical examination, vital signs, electrocardiogram, abdominal ultrasound, and both standard laboratory evaluations (hematology, chemistry, urinalysis) and special laboratory evaluations (thyroid function, glycosylated hemoglobin, carotene) were 
performed at baseline (week 0 , study day 1) and at the end of study (week 24). Vital signs were checked at weeks 4, 8, 12, 16, and 20, and standard laboratory evaluations were performed at weeks 4 and 12 . Adverse events were recorded during screening and washout, at baseline, and at each scheduled visit throughout the study period (weeks $4,8,12,16,20$, and 24). Injection sites were evaluated during the visit and then throughout the study period in daily diaries.

\section{Statistical Analysis}

Because of the low natural incidence of the carcinoid syndrome, only a limited number of patients were expected. Consequently, the study was designed to look at equivalence between the SC and LAR formulations of octreotide and not to detect statistically significant differences among the treatment groups. Although inferential testing of differences among groups was nevertheless performed as described in the following paragraph, conclusions were based on clinical rather than statistical findings.

Comparisons among treatment groups were analyzed using a pairwise Fisher's exact test for categorical efficacy variables (treatment response) and two models of a one-way analysis of variance (pairwise comparisons among three LAR doses, and SC $v$ each LAR dose) for continuous efficacy variables (number of daily stools and flushing episodes). A one-sample $t$ test was used to examine within-group change-from-screening and change-from-baseline comparisons for 5-HIAA. Demographic and baseline characteristics were examined for differences between groups using a $\chi^{2}$ test or Fisher's test for categorical variables and an analysis of variance for continuous variables. Descriptive summary statistics were provided for safety data.

\section{RESULTS}

\section{Patient Demographics and Clinical Characteristics}

Ninety-three patients were enrolled and randomized to receive octreotide LAR $10 \mathrm{mg}(\mathrm{n}=22), 20 \mathrm{mg}(\mathrm{n}=20)$, or $30 \mathrm{mg}(\mathrm{n}=25)$ or to continue SC octreotide every 8 hours $(n=26)$. These 93 patients constituted the intent-to-treat (ITT) population of patients who had a baseline assessment and at least one postbaseline efficacy assessment. The efficacy-assessable population comprised 79 patients who returned for at least the 20-week visit and evaluation, adhered to the schedules of laboratory sampling and diary completion, and met the criteria for symptom assessment during washout. Thirteen of the 93 patients discontinued before week 24; reasons for the discontinuations included consent withdrawal $(\mathrm{n}=2)$, failure to return for scheduled visits $(\mathrm{n}=4)$, adverse event $(\mathrm{n}=1)$, treatment failure $(\mathrm{n}=$ $2)$, death $(n=3)$, and unknown $(n=1)$. Since nine of the discontinuations occurred before week 20 and five patients completed the study but were later found not to have met the necessary inclusion criteria during the baseline period, the efficacy-assessable population was 79 patients.

Demographic and clinical characteristics of the four treatment groups were comparable except for age. The median age of patients in the 20-mg group was 7.1 to 11.5 years younger than that of any other group (Table 1).

Table 1. Baseline Patient Characteristics by Treatment Group

\begin{tabular}{|c|c|c|c|c|c|c|c|c|c|c|c|}
\hline \multirow[b]{3}{*}{ Variable } & \multirow{2}{*}{\multicolumn{2}{|c|}{ Total }} & \multirow{2}{*}{\multicolumn{2}{|c|}{ SC 0 ctreotide Every 8 Hours }} & \multicolumn{6}{|c|}{$O$ ctreotide LAR } & \multirow[b]{3}{*}{$P^{*}$} \\
\hline & & & & & \multicolumn{2}{|c|}{$10 \mathrm{mg}$} & \multicolumn{2}{|c|}{$20 \mathrm{mg}$} & \multicolumn{2}{|c|}{$30 \mathrm{mg}$} & \\
\hline & $\begin{array}{l}\text { No. of } \\
\text { Patients }\end{array}$ & $\%$ & $\begin{array}{l}\text { No. of } \\
\text { Patients }\end{array}$ & $\%$ & $\begin{array}{l}\text { No. of } \\
\text { Patients }\end{array}$ & $\%$ & $\begin{array}{l}\text { No. of } \\
\text { Patients }\end{array}$ & $\%$ & $\begin{array}{l}\text { No. of } \\
\text { Patients }\end{array}$ & $\%$ & \\
\hline No. of patients & 93 & & 26 & & 22 & & 20 & & 25 & & \\
\hline$<60$ years & 41 & 44.1 & 12 & 46.2 & 6 & 27.3 & 13 & 65.0 & 10 & 40.0 & .098 \\
\hline$>60$ years & 52 & 55.9 & 14 & 53.8 & 16 & 72.7 & 7 & 35.0 & 15 & 60.0 & \\
\hline \multicolumn{12}{|l|}{ Age, years } \\
\hline Mean & \multicolumn{2}{|c|}{59.5} & \multicolumn{2}{|c|}{59.4} & \multicolumn{2}{|c|}{61.4} & \multicolumn{2}{|c|}{54.0} & \multicolumn{2}{|c|}{63.0} & .039 \\
\hline Median & & & \multicolumn{2}{|c|}{63.7} & \multicolumn{2}{|c|}{62.3} & \multicolumn{2}{|c|}{55.2} & \multicolumn{2}{|c|}{66.7} & \\
\hline \multicolumn{12}{|l|}{ Sex } \\
\hline Male & 52 & 55.9 & 12 & 46.2 & 14 & 63.6 & 8 & 40.0 & 18 & 72.0 & .102 \\
\hline Female & 41 & 44.1 & 14 & 53.8 & 8 & 36.4 & 12 & 60.0 & 7 & 28.0 & \\
\hline \multicolumn{12}{|l|}{ Body weight, $\mathrm{kg}$} \\
\hline Mean & & & \multicolumn{2}{|c|}{77.3} & \multicolumn{2}{|c|}{77.0} & \multicolumn{2}{|c|}{75.5} & \multicolumn{2}{|c|}{77.2} & .979 \\
\hline Median & & & \multicolumn{2}{|c|}{76.6} & \multicolumn{2}{|c|}{76.9} & \multicolumn{2}{|c|}{78.0} & \multicolumn{2}{|c|}{81.7} & \\
\hline \multicolumn{12}{|l|}{ Disease duration, years } \\
\hline Mean & \multirow{2}{*}{\multicolumn{2}{|c|}{5.3}} & \multicolumn{2}{|c|}{4.8} & \multicolumn{2}{|c|}{$4.7 \dagger$} & & & 5.8 & & .783 \\
\hline Median & & & & & & & & & 4.7 & & \\
\hline Duration of SC octreotide, & & & & & & & & & & & \\
\hline Mean & & & & & & & & & 2.4 & & .138 \\
\hline Median & & & & & & & 1 & & 1.5 & & \\
\hline Mean daily SC dose, mg & & & & & & & & & 0.5 & & \\
\hline
\end{tabular}

*Between trea tment group difference.

†Data not available for two patients.

$\ddagger$ Dose information not available for one patient. 
Although disease burden was not determined, the median duration of disease ranged from 2.9 to 4.7 years for the different groups.

\section{Pharmacokinetics}

Mean trough octreotide levels for the octreotide LAR treatment groups are shown in Fig 1. Steady-state was reached by week 8 for the 20 -mg and 30-mg groups and week 12 for the 10-mg group (Fig 1). From week 8 through week 24, women and older patients ( $>60$ years of age) had higher mean and median levels of octreotide than men and younger patients, and the rate of rise to steady-state was slower for women than for men. Octreotide LAR administered every 4 weeks for 24 weeks resulted in octreotide trough concentrations that were dose-dependent and proportional to the dose. Mean steady-state octreotide levels were in the therapeutic range $(1,000$ to $4,000 \mathrm{pg} / \mathrm{mL})$ for carcinoid syndrome in all three octreotide LAR groups.

\section{Treatment Response}

The rates of treatment response at weeks 20 and 24 were similar across treatment groups for the efficacy-assessable population (Fig 2). Results were similar for the ITT population. No association was noted between the rates of complete treatment success and octreotide levels or between treatment response and sex or age.

\section{Need for Rescue}

Efficacy-assessable patients in the LAR groups required rescue medication earlier during treatment (about day 20) when compared with the SC group (day 59); the percentage

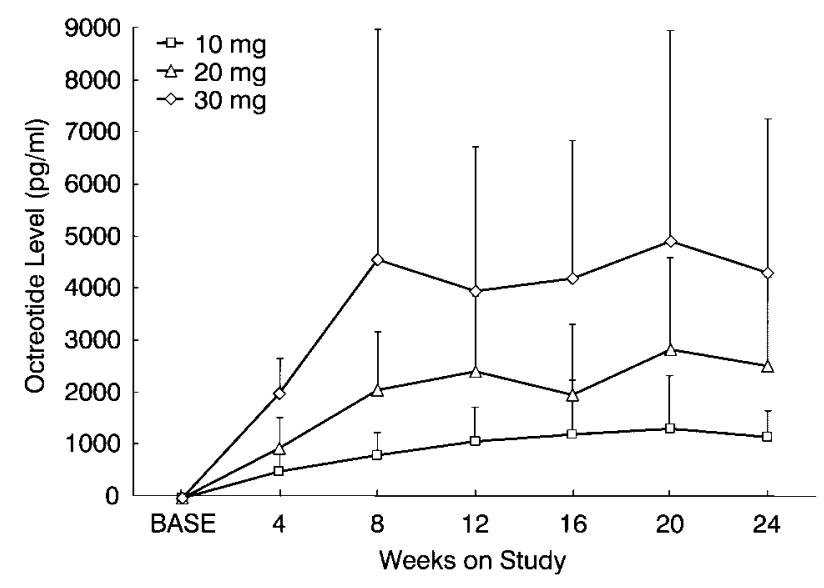

Fig 1. Mean serum octreotide concentration $(\mathrm{pg} / \mathrm{mL}$ ) (efficacy-assessable population) after intramuscular administration of octreotide LAR at $10 \mathrm{mg}$ ( $\mathrm{n}=16), 20 \mathrm{mg}(\mathrm{n}=13)$, or $30 \mathrm{mg}(\mathrm{n}=19)$ every 4 weeks. Baseline octreotide levels $>160 \mathrm{pg} / \mathrm{mL}$ were excluded from analysis. (Data were excluded from analysis for one patient in the $10-\mathrm{mg}$ group with an octreotide level of $0 \mathrm{pg} / \mathrm{mL}$ at week 24.)

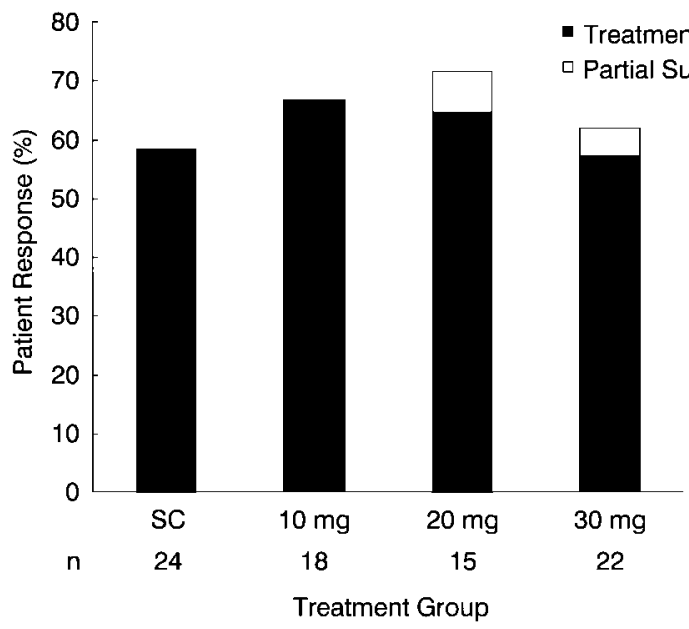

Fig 2. Percentage of patients (efficacy-assessable population) with treatment success and partial treatment success at week 20 and/ or week 24 by treatment group.

of patients requiring rescue was highest in the 10-mg LAR group (Fig 3). The need for rescue was similar in all four groups after week 16, but it was somewhat higher in the SC group at the end of the trial. Results were similar for the ITT population.

The SC patients who used supplementary rescue doses consistently required a higher median number of doses for each 4-week treatment interval than the patients in the LAR groups (Fig 4). Patients in the 10-mg LAR group used the smallest median number of rescue doses.

\section{Control of Diarrhea}

The median number of daily stools in both the efficacyassessable and ITT populations decreased significantly from baseline levels in all treatment groups and were similar

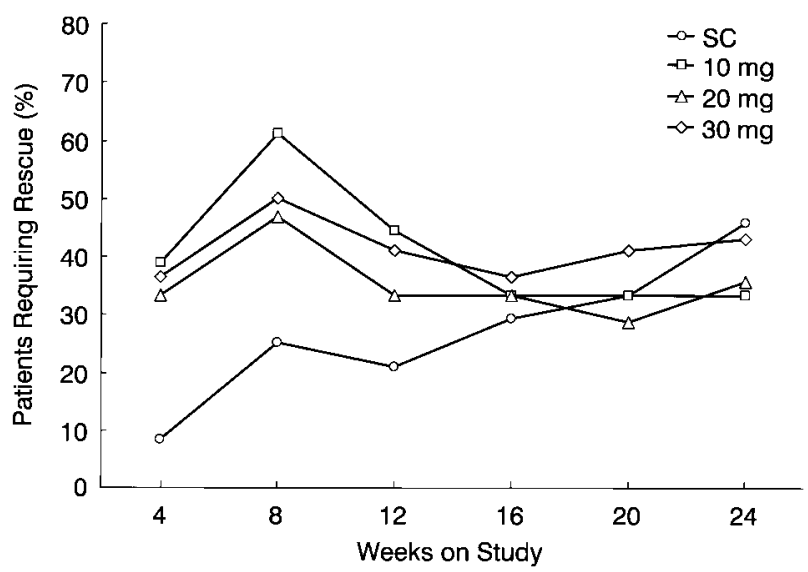

Fig 3. Percentage of patients (efficacy-assessable population) requiring rescue in each treatment group during the 24-week treatment period. 


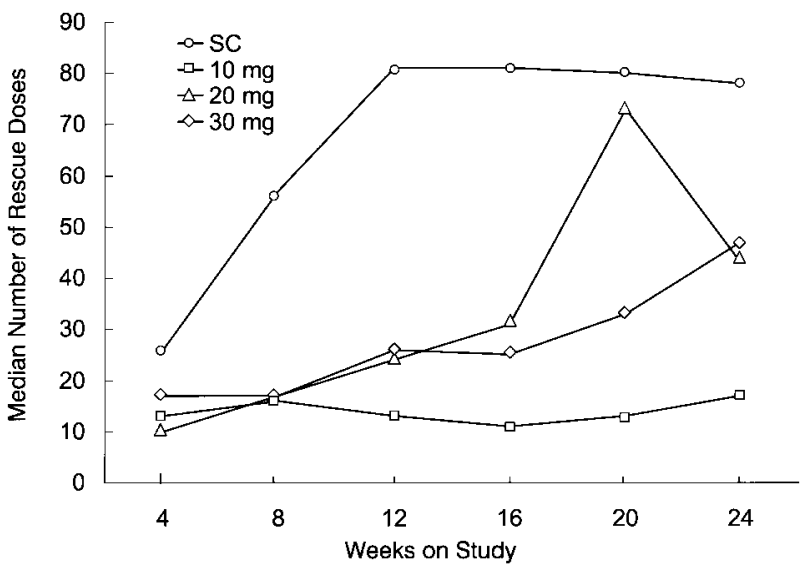

Fig 4. Median number of supplemental SC octreotide doses taken by patients (efficacy-assessable population) requiring rescue during the preceding 4-week interval by treatment group. The data for week 4 includes only those rescue doses taken after day 11.

across treatment groups (Fig 5). Occasional statistically significant, but not clinically relevant, differences across visits were noted within and between octreotide LAR treatment groups but not between LAR groups and the SC group.

\section{Control of Flushing}

The number of flushing episodes was higher for the 10-mg and 30-mg LAR groups than the SC group until week 8 and continued to be higher throughout the study in the 10 -mg group, although the differences were not statistically significant (Fig 6).

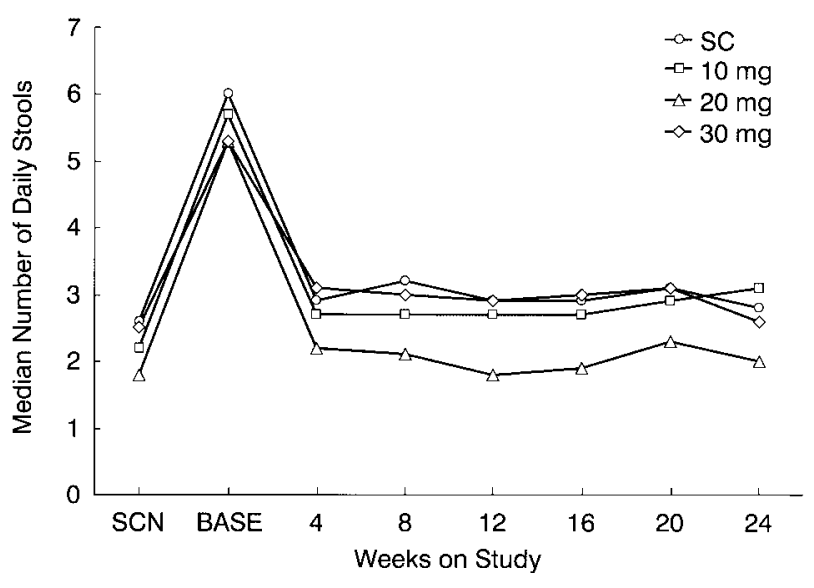

Fig 5. Median number of stools per day in 47 efficacy-assessable patients throughout screening (SCN), baseline (BASE), and the 24-week treatment period by treatment group.

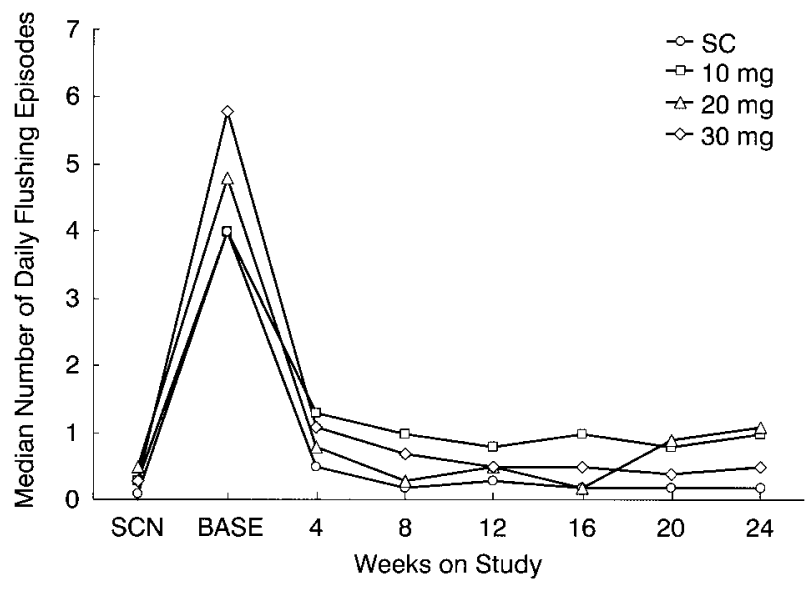

Fig 6. Median number of flushing episodes per day in the 33 efficacyassessable patients throughout screening (SCN), baseline (BASE), and the 24-week treatment period by treatment group.

\section{Urinary 5-HIAA Levels}

Twenty-four-hour urinary 5-HIAA data were available for 73 patients in the efficacy-assessable population. The use of 5-HIAA as a biochemical marker in this study was complicated by several factors, in particular, low patient compliance resulting in small patient numbers screened for 5-HIAA that prevented detection of any statistically significant differences between the SC group and LAR groups at most time points. In both the efficacy-assessable and ITT populations, the 20-mg LAR and SC doses most consistently reduced 24-hour urinary 5-HIAA to the controlled levels recorded during screening (Table 2). The different numbers

Table 2. Median and Range of 24-Hour Urinary Concentrations of 5-HIAA $(\mathrm{mg})$ in the Efficacy-Assessable Population Throughout Screening, Baseline, and End Point by Treatment Group

\begin{tabular}{|c|c|c|c|c|}
\hline & \multirow{2}{*}{$\begin{array}{c}\text { SC } \\
0 \text { ctreotide } \\
\text { Every } 8 \text { Hours }\end{array}$} & \multicolumn{3}{|c|}{ O ctreotide LAR } \\
\hline & & $10 \mathrm{mg}$ & $20 \mathrm{mg}$ & $30 \mathrm{mg}$ \\
\hline \multicolumn{5}{|l|}{ Screening* } \\
\hline N o. of Patients & 19 & 16 & 12 & 18 \\
\hline Median & 27.5 & 27.8 & 28.9 & 34.9 \\
\hline Range & $8.3-93.5$ & $2.3-652.7$ & $1.0-210.8$ & $1.1-495.0$ \\
\hline \multicolumn{5}{|l|}{ Baselinet } \\
\hline N o. of Patients & 20 & 14 & 13 & 14 \\
\hline Median & 48.5 & 72.7 & 52.6 & 83.0 \\
\hline Range & $12.3-223.4$ & $5.4-826.9$ & $3.1-513.3$ & $10.3-230.9$ \\
\hline \multicolumn{5}{|l|}{ End pointt } \\
\hline N o. of Patients & 21 & 18 & 14 & 20 \\
\hline Median & 35.4 & 68.9 & 24.0 & 77.2 \\
\hline Range & $0.1-240.0$ & $3.2-717.8$ & $2.8-223.3$ & $0.6-473.3$ \\
\hline
\end{tabular}

*Screening was defined as control of flushing and diarrhea with patient receiving SC octreotide every 8 hours.

†Baseline was defined as recurrence of carcinoid syndrome symptoms with patient not receiving SC octreotide every 8 hours.

$\ddagger$ End point defined as week 20 and/ or week 24 . 
of patients and the wide variability in the actual results reported here as median 24-hour urinary 5-HIAA levels reflect the low patient compliance in collecting and returning the urine samples for analysis. Nevertheless, there was no correspondence between 24-hour urinary 5-HIAA levels and octreotide levels.

\section{Safety}

Therapy was well tolerated in all four treatment groups. The majority of patients ( $84 \%$ to $95.4 \%$ ) reported adverse events, but most were mild to moderate in severity and were considered to be unrelated to therapy. No patient discontinued therapy because of a drug-related adverse event. The most frequently reported adverse events considered by the investigators to be possibly, probably, or definitely related to treatment with octreotide are listed in Table 3. The most common adverse events were gastrointestinal disorders, as is expected with octreotide treatment (abdominal pain, $\mathrm{n}=1$; flatulence, $\mathrm{n}=2$; nausea, $\mathrm{n}=3$; and steatorrhea, $\mathrm{n}=1$ ). Cholelithiasis was the only treatment-related adverse event to occur in all four groups; newly occurring gallbladder abnormalities detected by ultrasound included gallstones $(\mathrm{n}=5)$, sludge $(\mathrm{n}=2)$, and dilatation of the ductal system $(n=4)$. Seventeen patients experienced serious adverse events. All were considered by the investigators to have been unrelated to treatment, and only one (progressive carcinoid tumor) resulted in discontinuation. Three of these 17 patients died during the treatment phase of the study. Two deaths in the 30-mg LAR group and one in the 20-mg LAR group were considered unrelated (respiratory distress, pulmonary embolism) or unlikely related (hypoglycemic episodes with renal failure), respectively, to medication. No differences among the four treatment groups were observed with respect to vital signs, electrocardiograms, physical examinations, gallbladder ultrasound, or laboratory evaluations.

Table 3. N umber of Patients W ith Treatment-Related Adverse Events of Any Severity by Treatment Group

\begin{tabular}{lcccc}
\hline & $\begin{array}{c}\text { SC } \\
\text { A ctreotide } \\
(n=26)\end{array}$ & $\begin{array}{c}10 \mathrm{mg} \\
(\mathrm{n}=22)\end{array}$ & $\begin{array}{c}20 \mathrm{mg} \\
(\mathrm{n}=20)\end{array}$ & $\begin{array}{c}30 \mathrm{mg} \\
(\mathrm{n}=25)\end{array}$ \\
\cline { 3 - 5 } A pplication site disorder & 1 & 0 & 1 & 1 \\
A sthenia & 0 & 1 & 0 & 0 \\
Fever & 0 & 1 & 0 & 0 \\
Hypothyroidism & 0 & 0 & 1 & 0 \\
Abdominal pain & 1 & 0 & 0 & 0 \\
Flatulence & 1 & 0 & 0 & 1 \\
Nausea & 1 & 1 & 1 & 0 \\
Steatorrhea & 0 & 0 & 0 & 1 \\
Cholelithiasis & 2 & 1 & 1 & 1 \\
Rash & 0 & 0 & 2 & 0 \\
Taste perversion & 0 & 1 & 0 & 0 \\
Renal calculus & 0 & 0 & 0 & 1 \\
\hline
\end{tabular}

\section{DISCUSSIO N}

The efficacy of octreotide LAR $10 \mathrm{mg}, 20 \mathrm{mg}$, or $30 \mathrm{mg}$ administered once monthly was similar to that of multiple daily SC injections of octreotide in controlling the diarrhea and flushing associated with malignant carcinoid syndrome. Because of the time required to reach steady-state octreotide levels, as the drug is slowly released from the microspheres, patients crossing over to the LAR formulation need to continue to receive SC octreotide for approximately 2 weeks, and some individuals may need additional rescue SC octreotide for up to 2 to 3 months.

Suppression of urinary 5-HIAA levels in the 20-mg LAR group was virtually identical to that of the control group receiving SC octreotide, although the 10-mg dose of octreotide LAR was consistently the least effective. However, it should be noted that the 10-mg group also had the greatest increase in mean and median 5-HIAA concentrations during the washout period. Additionally, the determination of urinary 5-HIAA excretion may not be an ideal measure of outcome in carcinoid syndrome, as it does not seem to correspond with survival or to predict cardiovascular problems during surgery. ${ }^{17}$ Altered 5-HIAA levels can have other causes, including untreated malabsorption states and ingestion of serotonin-containing foods and certain drugs. ${ }^{8,12,18}$ Also, many foregut carcinoid tumors lack the enzyme necessary for efficient conversion of 5-hydroxytryptophan to 5-HIAA. ${ }^{12,14}$ Finally, patient numbers in each group were small. The 20-mg octreotide LAR dosage is recommended for initiation of treatment because of its overall apparent superiority: symptom control was reached faster, steadystate was achieved more rapidly compared with the 10-mg dose, less rescue treatment was required during the early period before steady-state was reached, and flushing was better controlled.

A 2-week trial period with SC octreotide is recommended for patients who have never been treated with SC octreotide. Initial treatment with SC octreotide will allow a determination to be made whether the patient responds to octreotide without significant adverse effects. Furthermore, because it takes several weeks after the first LAR injection for steadystate therapeutic levels of octreotide to be reached, it is recommended that all patients continue SC octreotide injections for the first 2 weeks after starting treatment with octreotide LAR.

Numerous trials have indicated that SC octreotide is well tolerated, with the principal side effects being injection site reactions and gastrointestinal complaints. ${ }^{19}$ Some side effects are transient and abate with continued treatment, which occurs with octreotide LAR treatment in patients with acromegaly. ${ }^{1,15,20}$ In this prospective randomized trial, the safety profile of octreotide LAR was similar to that of SC 
octreotide administered every 8 hours for the treatment of carcinoid syndrome. Reactions to the intramuscular injections were mild, and treatment-related adverse events were infrequent and generally mild to moderate in severity. The incidence of gallstone development, which was low, was probably due at least in part to the fact that patients had previously received SC octreotide every 8 hours for a median of 2.8 years. It is known that long-term octreotide treatment is associated with an increased prevalence of gallbladder sludge and gallstones in approximately $20 \%$ to $30 \%$ of patients. ${ }^{15,19,21}$ Within the octreotide LAR dose range used (10 to $30 \mathrm{mg}$ ), no dose relationship was observed in the incidence or severity of adverse events.
In summary, octreotide LAR provides efficacy comparable to SC octreotide with a similar safety profile. Moreover, the availability of a once-monthly injection may increase the number of patients willing to undergo treatment. Thus, octreotide LAR is an alternative to SC octreotide with the potential to improve patient compliance and satisfaction with therapy, leading to successful clinical control of the carcinoid syndrome.

\section{ACKN O W LEDG MENT}

We are indebted to Louis Boyajy, $\mathrm{PhD}$, for protocol design and Jenny Darcy, Pamela Dumas, RN, Kristen Hargraves, RN, Lisa Martin, RN, Carl McWatters, Sally Pratt, RN, Lynn Russ, RN, Karen Small, RN, Alison Sugarman, and Sheilah Winn, RN, for project management and coordination.

\section{REFEREN CES}

1. Kvols LK: Metastatic carcinoid tumors and the malignant carcinoid syndrome. Ann NY Acad Sci 733:464-470, 1994

2. Roberts LJ II, Anthony LB, Oates JA: Disorders of vasodilator hormones: The carcinoid syndrome and mastocytosis, in Wilson JD, Foster DW (eds): Williams Textbook of Endocrinology (ed 9). Philadelphia, PA, WB Saunders, 1998, pp 1619-1634

3. Redfern J, O'Dorisio TM: Gastrointestinal hormones and carcinoid syndrome, in Felig P, Baxter JD, Frohman LA (eds): Endocrinology and Metabolism (ed 3). New York, NY, McGraw-Hill, 1995, pp 1675-1700

4. Newton JN, Swerdlow AJ, dos Santos Silva IM, et al: The epidemiology of carcinoid tumours in England and Scotland. $\mathrm{Br} \mathrm{J}$ Cancer 70:939-942, 1994

5. Godwin JD II: Carcinoid tumors: An analysis of 2837 cases. Cancer 36:560-569, 1975

6. Surveillance, Epidemiology, and End-Results (SEER): Cancer Statistics Review. Bethesda, MD, U.S. Department of Health and Human Services, Public Health Institute, National Institutes of Health, National Cancer Institute, 1993

7. Modlin IM, Sandor A: An analysis of 8305 cases of carcinoid tumors. Cancer 79:813-829, 1997

8. Kaplan LM: Endocrine tumors of the gastrointestinal tract and pancreas, in Isselbacher KJ, Braunwald E, Wilson JD, et al (eds): Harrison's Principles of Internal Medicine (ed 13). New York, NY, McGraw-Hill, 1994, pp 1535-1542

9. Janmohamed S, Bloom SR: Carcinoid tumours. Postgrad Med J 73:207-214, 1997

10. Gorden P, Comis RJ, Maton PN, et al: Somatostatin and somatostatin analogue (SMS 201-995) in treatment of hormonesecreting tumors of the pituitary and gastrointestinal tract and nonneoplastic diseases of the gut. Ann Intern Med 110:35-50, 1989
11. Kvols LK, Moertel CG, O'Connell MJ, et al: Treatment of the malignant carcinoid syndrome: Evaluation of a long-acting somatostatin analogue. N Engl J Med 315:663-666, 1986

12. Norton JA, Levin B, Jensen RT: Cancer of the endocrine system, in DeVita VT, Hellman S, Rosenberg SA (eds): Cancer: Principles and Practice of Oncology (ed 4). Philadelphia, PA, Lippincott, 1993, pp 1333-1435

13. Buchanan KD: Effects of Sandostatin on neuroendocrine tumours of the gastrointestinal system. Recent Results Cancer Res 129:45-55, 1993

14. Creutzfeldt W: Carcinoid tumors: Development of our knowledge. World J Surg 20:126-131, 1996

15. Lancranjan I, Bruns C, Grass P, et al: Sandostatin $\operatorname{LAR}^{\circledR}$ : A promising therapeutic tool in the management of acromegalic patients. Metabolism 45:67-71, 1996 (suppl 1)

16. Lancranjan I, Bruns C, Grass $P$, et al: Sandostatin $\mathrm{LAR}^{\circledR}$ : Pharmacokinetics, pharmacodynamics, efficacy, and tolerability in acromegalic patients. Metabolism 44:18-26, 1995

17. Bax ND, Woods HF, Batchelor A, et al: Octreotide therapy in carcinoid disease. Anticancer Drugs 7:17-22, 1996 (suppl 1)

18. Creutzfeldt W, Stöckmann F: Carcinoids and carcinoid syndrome. Am J Med 82:4-16, 1987 (suppl 5B)

19. Harris AG, O'Dorisio TM, Woltering EA, et al: Consensus statement: octreotide dose titration in secretory diarrhea: Diarrhea Management Consensus Development Panel. Dig Dis Sci 40:1464-1473, 1995

20. Battershill PE, Clissold SP: Octreotide: A review of its pharmacodynamic and pharmacokinetic properties, and therapeutic potential in conditions associated with excessive peptide secretion. Drugs 38:658702,1989

21. Plockinger U, Dienemann D, Quabbe HJ: Gastrointestinal side effects of octreotide during long term treatment of acromegaly. J Clin Endocrinol Metab 71:1658-1662, 1990 